
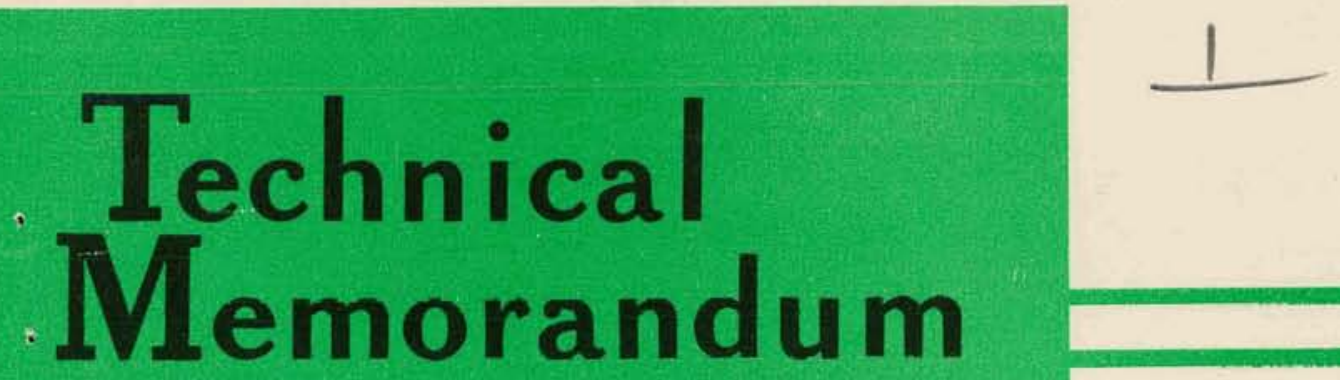

$\operatorname{AECU}-3224$

$151-55-51$

\title{
THICKNESS VIBRATIONS IN LONG RODS OF BARIUM TITANATE
}

C. V. Stephenson - 5133

LEGAL NOTICE

This report was prepared as an account of Government sponsored work. Neither the United States, nor the Commission, nor any person acting on behalf of the Commission:

A. Makes any warranty or representation, express or implied, with respect to the accuracy, completeness, or usefulness of the information contained in this report, or that the use of any information, apparatus, method, or process disclosed in this report may not infringe privately owned rights; or

B. Assumes any liabilities with respect to the use of, or for damages resulting from the use of any information, apparatus, method, or process disclosed in this report.

As used in the above, "person acting on behalf of the Commission" includes any employee or contractor of the Commission to the extent that such employee or contractor prepares, handles or distributes, or provides access to, any information pursuant to his employment or contract with the Commission.

\section{ABSTRACT}

A theoretical analysis of the thickness vibrations in long thin rods of barium titanate is presented. The theoretical results are checked experimentally and their meaning discussed.

Case No. 590

Photostat Price $\$ 3,30$

Microfilm Price $\$ 2.40$

Available from the

Office of Technical Services

Department of Commerce

Washington 25, D. C. 


\section{DISCLAIMER}

This report was prepared as an account of work sponsored by an agency of the United States Government. Neither the United States Government nor any agency Thereof, nor any of their employees, makes any warranty, express or implied, or assumes any legal liability or responsibility for the accuracy, completeness, or usefulness of any information, apparatus, product, or process disclosed, or represents that its use would not infringe privately owned rights. Reference herein to any specific commercial product, process, or service by trade name, trademark, manufacturer, or otherwise does not necessarily constitute or imply its endorsement, recommendation, or favoring by the United States Government or any agency thereof. The views and opinions of authors expressed herein do not necessarily state or reflect those of the United States Government or any agency thereof. 


\section{DISCLAIMER}

Portions of this document may be illegible in electronic image products. Images are produced from the best available original document. 


\section{2 \\ THICKNESS VIBRATIONS IN LONG RODS OF \\ BARIUM TITANATE}

\section{INTRODUCTION}

From time to time the question arises as to how one can best measure the piezoelectric constant $d_{33}$ in barium titanate. One common method of obtaining values for this constant is an indirect one involving the measurement of the hydrostatic $d$-constant, $d_{h}$, which is theoretically related to $d_{33}$ by the equation $d_{h}=d_{33}+2 d_{31}$. The piezoelectric constant $d_{31}$ is easily obtained by resonant and anti-resonant measurement of the longitudinal mode of vibration in long thin rods or the radial mode of vibration in thin discs. The hydrostatic measurement consists of subjecting the element to a sinusoidally varying hydrostatic pressure and comparing its output with the output of a crystal of supposedly known characteristics. This is a rather involved measurement and is probably more subject to error than the resonant-anti-resonant type of measurement. It is possible to measure $d_{33}$ by resonant and anti-resonant measurement of the thickness mode of vibration in long thin rods, although extreme precautions must be taken to avoid shunt capacity across the element during the measurement. Several authors have acknowledged the possibility of making this type of measurement, but have avoided a theoretical discussion of this mode of vibration saying that it is somewhat more involved than the usual modes of interest. Van Dyke ${ }^{1}$ has discussed this mode of vibration but his development is thought to be incorrect because he failed to allow for the variation of the electric field through the element. This memorandum presents a theoretical discussion of thickness vibrations and points out how the measurement of resonant and anti-res onant frequencies are pertinent in the calculation of $d_{33}$. It will be assumed that the reader is familiar with the type of development which is to be presented, at least for the relatively simple case of the longitudinal mode. Those who are not familiar with the development and wish to study further are referred to Mason's book ${ }^{2}$, particularly Chapters 3 and 5 . At the same time it is hoped that those who desire only to make use of the results will be able to read through the necessary mathematics with a minimum of difficulty.

${ }^{1}$ K. S. Van Dyke, Proc. I. R. E., 16, 742(1928)

"Piezoelectric Crystals and Their Application to Ultrasonics", W. P. Mason

(New York, D. Van Nostrand Co., Inc., 1950) 
The type of element with which we will be concerned is shown in Figure 1. According to convention, we will take the thickness in the $z$-direction and designate this dimension by $l_{t}$.

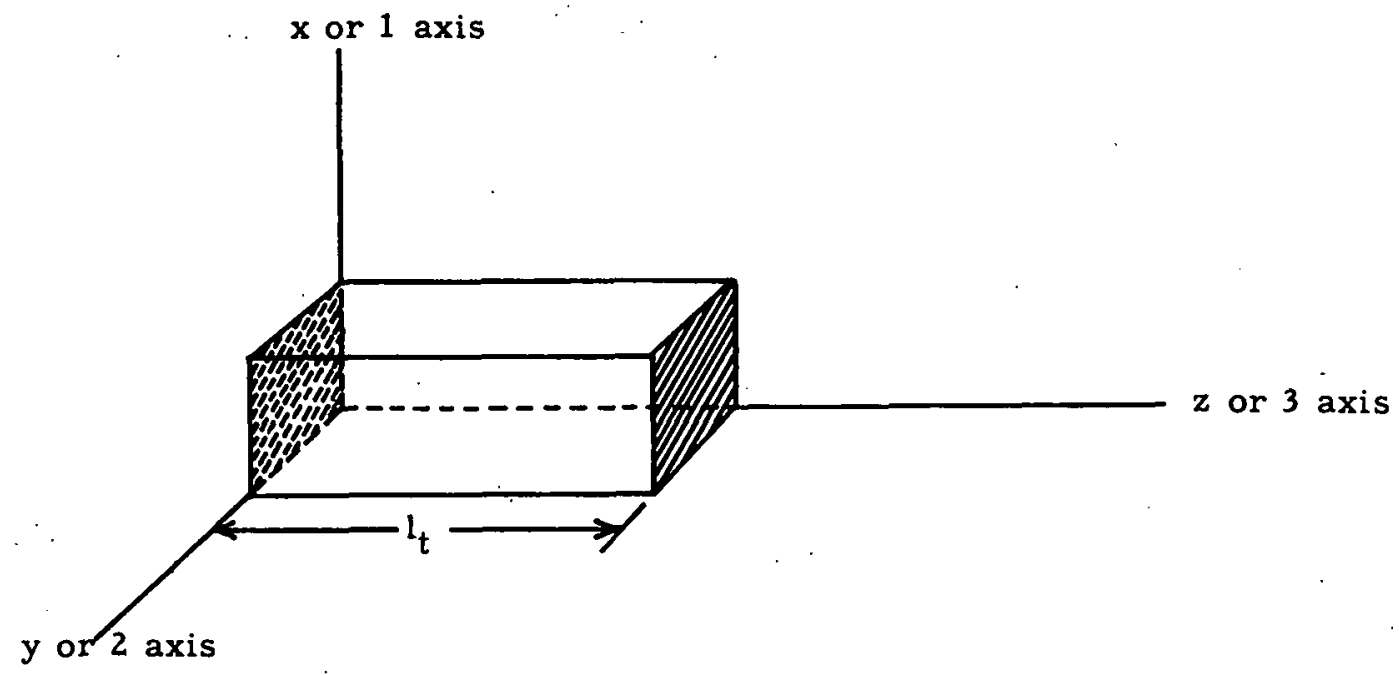

Fig. 1 -- Long Thin Rod Used for Thickness Vibrations

The other two dimensions of the element are small compared with $t_{t}$. The faces perpendicular to the $z$-axis are plated and a field $E_{3}$ is applied to these faces.

To begin with, we will need some force equations and piezoelectric relations which will not be developed. For the development of these equations, the reader is referred to the excellent presentation in Mason's book.

We will begin with the following force relations:

$$
\begin{aligned}
& F_{x}=p \frac{\partial^{2} \xi}{\partial t^{2}} d x d y d z=\left(\frac{\partial T_{1}}{\partial x}+\frac{\partial T_{6}}{\partial y}+\frac{\partial T_{5}}{\partial z}\right) d x d y d z \\
& F_{y}=\rho \frac{\partial^{2} \eta}{\partial t^{2}} d x d y d z=\left(\frac{\partial T_{6}}{\partial x}+\frac{\partial T_{2}}{\partial y}+\frac{\partial T_{4}}{\partial z}\right) d x d y d z \\
& F_{z}=\rho \frac{\partial^{2} \zeta}{\partial t^{2}} d x d y d z=\left(\frac{\partial T_{5}}{\partial x}+\frac{\partial T_{4}}{\partial y}+\frac{\partial T_{3}}{\partial z}\right) d x d y d z
\end{aligned}
$$

where the $F^{\prime} s$ are force components at a point in the material, the $T^{\prime} s$ are stress components, $\rho$ is the density and $\xi, \eta$, and $\zeta$ are mechanical displacements in the 
$x ; y$ and $z$ directions. These relations are derived from geometrical considerations of the elementary cube and are independent of what the stress-strain behavior of the material may be. We shall also need Mason's relations 3.58 which are derived from thermodynamic considerations. Using the convention that a repeated suffix indicates a. summation, these may be written:

$$
\begin{aligned}
& s_{i}=s_{i j}^{E} T_{j}+d_{m i} E_{m} \\
& \delta_{n}=d_{n j} T_{j}+\frac{{ }_{n m}}{4 \pi} E_{m}
\end{aligned}
$$

In these equations S's are strains, 'E's are electric fields, $\delta$ 's are electrical displacements divided by $4 \pi, s_{i j}^{E}$ 's are elastic compliances at constant field, $\epsilon_{n m}^{T}$ 's are dielectric constants at constant stress and $\mathrm{d}_{\mathrm{mi}}{ }^{\prime}$ s. are piezoelectric constants:

For the case under consideration, we will assume that there are no forces on the sides parallel to the thickness. Since the dimensions in these directions are assumed small, the change in stress through the element in these directions will be negligible and since they are zero at the surface, they will be assumed to be zero through the element so that we will say $T_{1}=T_{2}=T_{4}=T_{5}=T_{6}=0$. Also since we will apply an electric field only in the $z$ direction, $E_{1}=E_{2}=0$. The piezoelectric equations now reduce to:

$$
\begin{aligned}
& S_{i}=s_{i 3}^{E} T_{3}+d_{3 i} E_{3} \\
& \delta_{n}=d_{n 3} T_{3}+\frac{\epsilon}{43} E_{3}
\end{aligned}
$$

In particular $T_{3}$ may be specified by the single strain $S_{3}=\frac{\partial S}{\partial z}$ and the only charge of interest is in the $\mathrm{z}$-direction, hence, the equations of interest in the present case are:

$$
\begin{aligned}
& s_{3}=s_{33}^{E} T_{3}+d_{33} E_{3} \\
& \delta_{3}=d_{33} T_{3}+\frac{\epsilon_{33}^{T}}{4 \pi} E_{3}=\left[\frac{\epsilon_{33}^{T}}{4 \pi}-\frac{d_{33}^{2}}{s_{33}^{E}}\right] E_{3}+\frac{d_{33}}{s_{33}} s_{3}
\end{aligned}
$$

Also, the force equation becomes

$$
F_{3}=\rho \frac{\partial^{2} \zeta}{\partial t^{2}} d x d y d z=\frac{\partial T_{3}}{\partial z} d x d y d z
$$

or

$$
p \frac{\partial^{2} \zeta}{\partial t^{2}}=\frac{\partial T_{3}}{\partial z}
$$


At this time it would probably be instructive to stop and compare these last equations with the corresponding equations obtained for the case of longitudinal vibrations. The longitudinal mode is obtained in an element similar to the one shown in Figure 1 except that the electrodes are applied to two of the long faces. Also there is the convention that the field is applied in the $z$ direction so that there is an additional rotation of axes. The waves of rarefaction and compression are assumed to be plane waves traveling along the length of the element except that the direction of travel is perpendicular to the applied field in the longitudinal case, whereas, it is parallel to the field in the thickness mode. The corresponding equations for longitudinal are:

$$
\begin{aligned}
& s_{1}=s_{11}^{E} T_{1}+d_{31} E_{3} \\
& \delta_{3}=\left[\frac{\epsilon_{33}^{T}}{4 \pi}=\frac{d_{31}^{2}}{s_{11}^{E}}\right] E_{3}+\frac{d_{31}}{s_{11}^{E}} s_{1} \\
& \rho \frac{\partial^{2} \xi}{\partial t^{2}}=\frac{\partial T_{1}}{\partial x}
\end{aligned}
$$

where $S_{1}$ and $T_{1}$ are strain and stress components perpendicular to the direction of the applied field $E_{3}$. Although these equations are quite similar to those for the thickness mode, the fact that the stress and strain $T_{3}$ and $S_{3}$ in the thickness equations are parallel to the field $\mathrm{E}_{3}$, is sufficient to complicate the boundary conditions of the problem considerably. This can be seen as follows: the further development of these equations require that one solve for the strain $S_{1}$ as a function of the coordinates of the sys tem. This will be obtained as the solution of a wave equation but there will be some arbitrariness to the solution in terms of two arbitrary constants. In the longitudinal case, one can return to the equation for $S_{1}$ and apply the condition that for a free element $T_{1}$ is zero at both ends. Furthermore, since the $z$ faces are equipotential surfaces and since $S_{1}$ is a function of $x$ only, $E_{3}$ will be a function of neither $x$ or $z$, that is, it is a constant. Thus, the boundary conditions are applied with relative ease. However, for the thickness mode $S_{3}$ is a function of $z$. which requires that $E_{3}$ also be a function of $\mathrm{z}$. One then must look for other conditions to apply. The argument that will be used is that lines of $\delta_{3}$ must end on free charges and since there can be no free charge in the interior of the element, then it must be that $\partial \delta_{3} / \partial z=0$. The nature of this condition means that only half of the arbitrariness of $S_{3}$ can be removed, the other half being carried as an undetermined constant. Fortunately, in eventually arriving at a usuable expression, this undetermined constant will appear as a multiplier in both numerator and denominator of a fraction and will cancel. Having thus seen where the complications arise, we now return to the development of the thickness mode.

Defining the thickness clamped dielectric constant by the expression

$$
\frac{\epsilon_{33}^{T C}}{4 \pi}=\frac{\epsilon_{33}^{T}}{4 \pi}-\frac{d_{33}^{2}}{s_{33}^{E}}
$$


one gets the expression

$$
-\frac{\partial E_{3}}{\partial z}=\frac{d_{33}}{\frac{\epsilon_{33}^{T C}}{4 \pi} s_{33}^{E}} \frac{\partial S_{3}}{\partial z}=\frac{d_{33}}{\frac{\epsilon_{C}}{4 \pi} s_{33}^{E}} \frac{\partial^{2} \zeta}{\partial z}
$$

so that we arrive at the wave equation

$$
\begin{aligned}
\rho \frac{\partial^{2} \zeta}{\partial t^{2}} & =\frac{\partial T_{3}}{\partial z}=\frac{1}{s_{33}^{E}} \frac{\partial^{2} \zeta}{\partial z^{2}}-\frac{d_{33}}{s_{33}^{E}} \frac{\partial E_{3}}{\partial z} \\
& =\frac{1}{s_{33}^{E}}\left(1+\frac{d_{33}^{2}}{\frac{\epsilon_{33}^{T C}}{4 \pi}} s_{33}^{E}\right) \frac{\partial^{2} \zeta}{\partial z^{2}}=Y_{0} \frac{\partial^{2} \zeta}{\partial z^{2}}
\end{aligned}
$$

where $Y_{0}=\frac{1}{s_{33}}\left(1+\frac{d_{33}^{2}}{\frac{\epsilon_{33}^{T C}}{4 \pi} s_{33}^{E}}\right)$ can be thought of as an effective Young's modulus.

This equation has the solution $\zeta=\zeta_{0} e^{j \omega t}$ where $\zeta_{0}=A \sin \frac{\omega z}{v}+B \cos \frac{\omega z}{v}$ and $v=\sqrt{\frac{Y_{o}}{\rho}}$. For a free element, $T_{3}=0$ at the surfaces $z=0$ and $z=t_{t}$ so that,

neglecting for the moment the time dependency, $S_{3}=\frac{\partial \zeta}{\partial z}=\frac{\omega}{v}\left(A \cos \frac{\omega z}{v}-B \sin \frac{\omega z}{v}\right)=$ $\mathrm{d}_{33} \mathrm{E}_{3}$ at these two boundaries.

This gives the two conditions $\frac{\omega A}{v}=d_{33} E_{3}(0)$ or $A=\frac{v}{\omega} d_{33} E_{3}(0)$ and $\frac{\omega}{v} A \cos \frac{\omega l_{t}}{v}-B \sin \frac{\omega l_{t}}{v}=d_{33} E_{3}\left(I_{t}\right)=d_{33} E_{3}(0)$ since $E_{3}\left(l_{t}\right)=E_{3}(0)$.

This gives for the other constant

$$
-B=\frac{\frac{v}{\omega} d_{33} E_{3}(0)-\frac{v}{\omega} d_{33} E_{3}(0) \cos \frac{\omega l}{y}}{\vdots \sin \frac{\omega l}{v}}=\frac{v}{\omega} d_{33} E_{3}(0) \frac{1-\cos \frac{\omega l_{t}}{v}}{\sin \frac{\omega l}{v}}
$$


so that

$$
S_{3}=d_{33} E_{3}(0)\left[\cos \frac{\omega z}{v}+\frac{1-\cos \frac{\omega l}{v}}{\sin \frac{\omega l}{v}} \sin \frac{\omega z}{v}\right]
$$

Now if $\frac{\partial \delta_{3}}{\partial z}=0$ then $\frac{\partial E_{3}}{\partial z}=-\frac{d_{33}}{\frac{\epsilon_{33}^{T C}}{4 \pi} s_{33}^{E}} \frac{\partial S_{3}}{\partial z}$ or $E_{3}=-\frac{d_{33}}{\frac{\epsilon_{33}^{T C}}{4 \pi} s_{33}^{E}} S_{3}+$ constant.

At $z=0$ this constant is found to be; constant $=E_{3}(0)+\frac{d_{33}^{2}}{\frac{T C}{4 \pi}} s_{33}^{E} E_{3}(0)=\frac{\epsilon_{33}^{T}}{\epsilon_{33}^{T C}} E_{3}(0)$

thus $E_{3}=-\frac{d_{33}^{2}}{\frac{\epsilon_{33}^{T C}}{4 \pi} s_{33}^{E}} E_{3}(0)\left[\cos \frac{\omega z}{v}+\frac{1-\cos \frac{\omega l}{v}}{\sin \frac{\omega l}{v}} \sin \frac{\omega z}{v}\right]+\frac{\epsilon_{33}^{T}}{\epsilon_{33}^{T C}} E_{3}(0)$

and also $\delta_{3}=\frac{\epsilon_{33}^{T C}}{4 \pi} E_{3}+\frac{d_{33}}{s_{33}^{E}} s_{3}=\frac{\epsilon_{33}^{T}}{4 \pi} E_{3}(0)$

The voltage across the element is found to be

$$
\begin{aligned}
& V=\int_{0}^{l_{t}} E_{3} d z=\frac{\frac{33}{T C}}{\epsilon_{33}^{T C}} E_{3}(0) l_{t}-\frac{d_{33}^{2} E_{3}(0)}{\frac{\epsilon_{33}^{T C}}{4 \pi} s_{33}} \frac{v}{\omega}\left[\sin \frac{\omega z}{v}-\frac{1-\cos \frac{\omega t}{v}}{\sin \frac{\omega l t}{v}} \cos \frac{\omega z}{v}\right]_{0}^{l_{t}} \\
& =\frac{\epsilon_{33}^{T}}{\epsilon_{33}^{T C}} E_{3}(0) l_{t}-\frac{d_{33}^{2}}{\frac{\epsilon_{33}^{T C}}{4 \pi} s_{33}^{E}} E_{3}(0) 1_{t} \cdot \frac{2 v}{\omega L_{t}} \tan \frac{\omega l_{t}}{2 v} \\
& =\frac{E_{3}(0) i_{t}}{\epsilon_{33}^{T C}}\left(\epsilon_{33}^{T}-\frac{4 \pi d_{33}^{2}}{s_{33}^{E}} \frac{\tan \frac{\omega l_{t}}{2 v}}{\frac{\omega l_{t}}{2 v}}\right)
\end{aligned}
$$


The equations for $\delta_{3}$ and $\mathrm{V}$ should, of course, be multiplied by the time dependent term $e^{j \omega t}$.

The current to the element is given by

$$
I=j \omega A \delta_{3}=j \omega A \frac{\epsilon_{33}^{T}}{4 \pi} \quad E_{3}(0),
$$

where $A$ is now the area of the electroded face of the element, so that the admittance of the element is

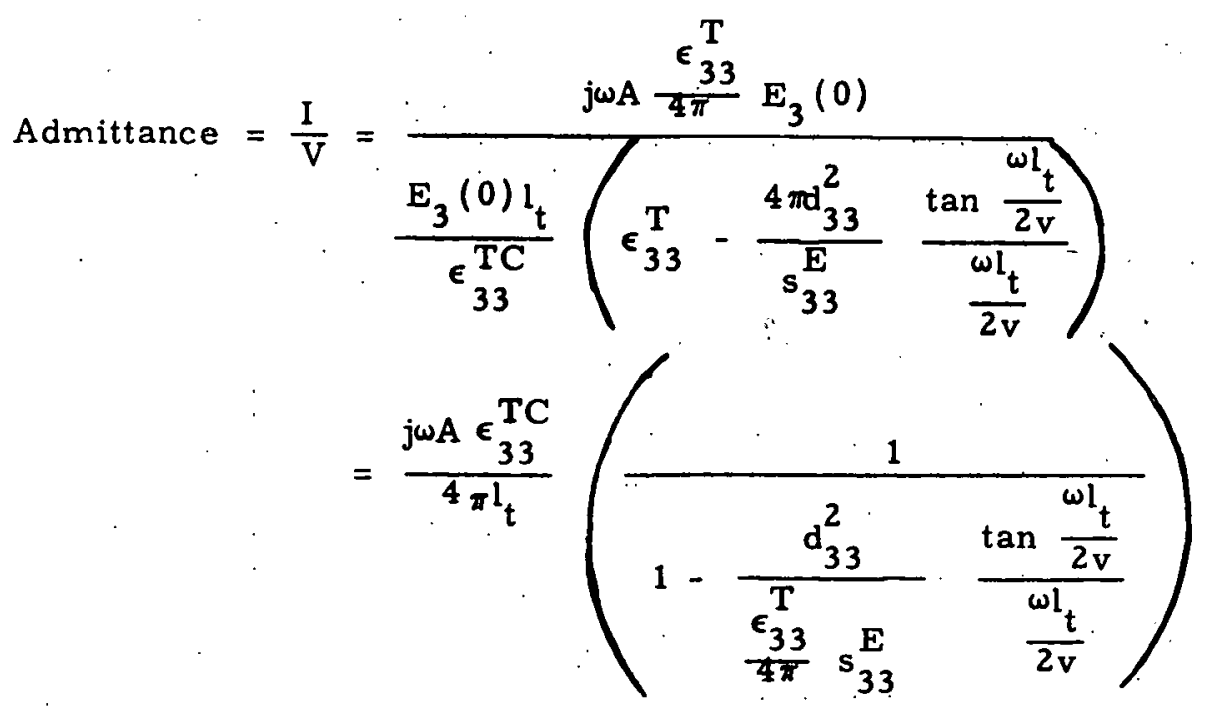

At mechanical resonance $\frac{{ }^{\omega} A t}{2 v}=\frac{\pi}{2}$ or Admittance $=0$ which corresponds to electrical anti-resonance. At electrical resonance Admittance $=\infty$ which happens when

$$
\frac{d_{33}^{2}}{\frac{\epsilon_{33}^{T}}{4 \pi} s_{33}^{E}} \frac{\tan \frac{\omega_{R} l}{2 v}}{\frac{\omega_{R} l}{2 v}}=1
$$

Now from the definition of the coupling coefficient $k_{33}^{2}=\frac{d_{33}^{2}}{\frac{\epsilon_{33}^{T}}{4 \pi} s_{33}^{E}}$

so that this condition becomes $\frac{\omega_{R} l_{t}}{2 v} \cot \frac{\omega_{R} l_{t}}{2 v}=k_{33}^{2} \cdot$ Also $v^{2}=\frac{1}{\rho s_{33}^{E}\left(1-k_{33}^{2}\right)}$. 
Since

$$
\cot \frac{\omega_{R} l_{t}}{2 v}=\cot \left(\frac{\omega_{A} l_{t}}{2 v}-\frac{\Delta \omega l_{t}}{2 v}\right)=\cot \left(\frac{\pi}{2}-\frac{\Delta \omega l_{t}}{2 v}\right)=\tan \frac{\Delta \omega l_{t}}{2 v} \text {, where } \Delta \omega=\omega_{A}-\omega_{R^{\prime}}
$$

we get $\frac{\omega_{R} l_{t}}{2 v} \cot \frac{\omega_{R} l_{t}}{2 v}=\left(\frac{\pi}{2}-\frac{\Delta \omega l_{t}}{2 v}\right) \tan \frac{\Delta \omega l_{t}}{2 v}=k_{33}^{2}$

Expanding $\tan x=x+\frac{1}{3} x^{3}+\frac{2}{15} x^{5} \cdots$

this becomes

$$
\left(\frac{\pi}{2}-\frac{\Delta \omega l_{t}}{2 v}\right)\left[\frac{\Delta \omega l_{t}}{2 v}+\frac{1}{3}\left(\frac{\Delta \omega l_{t}}{2 v}\right)^{3}+\cdots\right]=k_{33}^{2}
$$

so that to a first approximation

$$
\mathbf{k}_{33}^{2}=\frac{\pi}{2} \frac{\Delta \omega l_{t}}{2 v}=\frac{\pi^{2}}{4} \frac{\Delta \omega}{\omega_{A}}=\frac{\pi^{2}}{4} \frac{\Delta f}{f_{A}} .
$$

\section{DISCUSSION}

The results of the preceding section show that the anti-resonant frequency of the thickness mode of vibration plays the same part as the resonant frequency of most other modes of vibration and is controlled by the elastic properties of the material in the same way that the elastic constants control the resonant frequency in the other modes. This seemed strange and it was felt that some verification was needed. For that reason the following experiment was devised.

As in the other modes of vibration, the theory allows only for the odd harmonics, the even harmonics being absent. However, the above theory predicts that the anti-resonant frequency of the third harmonic should be exactly three times the anti-resonant frequency of the first harmonic, whereas, the theories of the other modes of vibration predict this type of relationship between resonant frequencies. This suggested a way of checking the validity of the assertion that the anti-resonant frequency is controlled by the elastic properties of the material and hence giving at least a left-handed check of the theory itself. The check was simply to compare the ratios of the resonant and anti-resonant erequencies for the first and third harmonics and to see which was equal to three. There was one difficulty. The theory assumed an element very long compared to its width. Since third harmonic measurement cut the effective length of the element to $1 / 3$ of its actual length, the finite length-to-width ratio would probably be apparent. To determine the effect of the length-to-width ratio, three different sized elements were used all having a length of $1^{\prime \prime}$, but having three different widths - $1 / 8^{\prime \prime}, 3 / 16^{\prime \prime}$ and $1 / 4^{\prime \prime}$. 


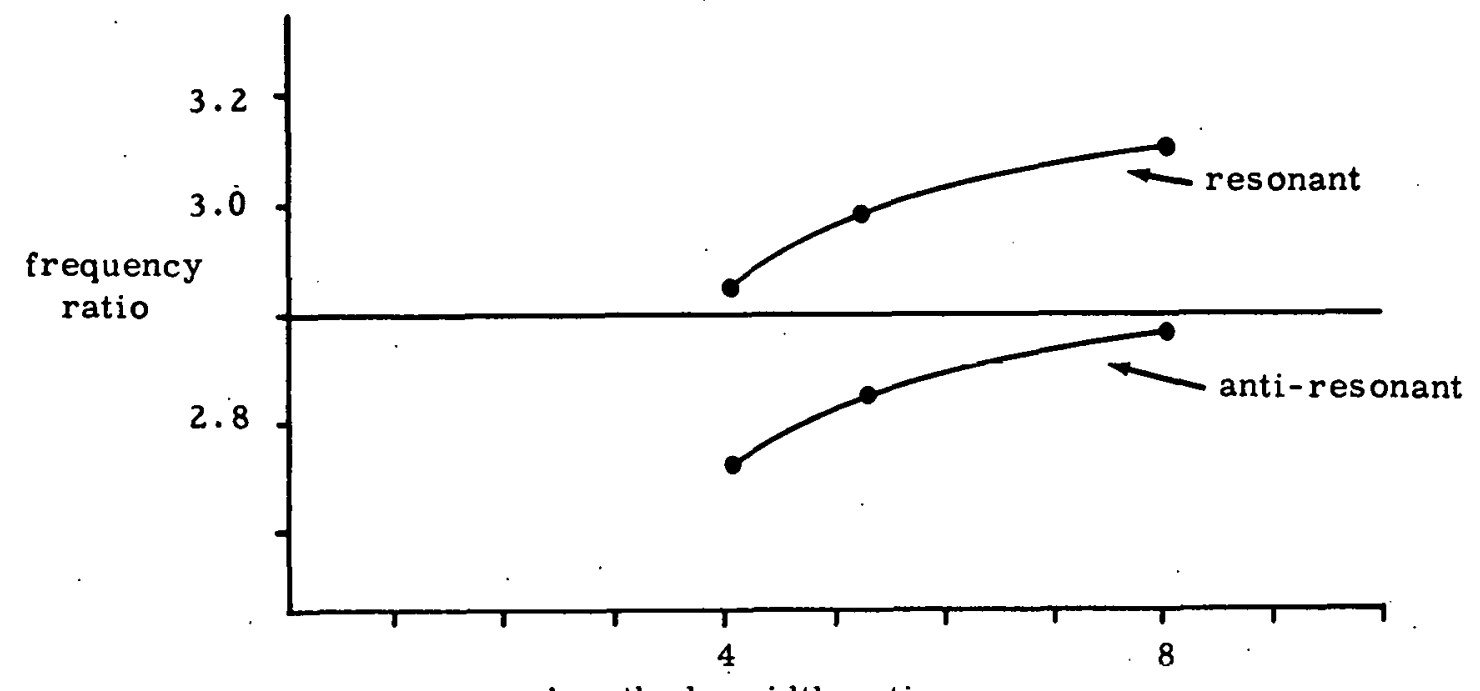

length-do-width ratio

Fig. 2 -- Plot of ratio of resonant and anti-resonant frequencies of first and third harmonic vers us length-to-width ratio.

Figure 2 shows the result of these measurements. In this figure the ratio of frequencies of the resonant and anti-resonant condition for the first and third harmonic are plotted against the length-to-width ratio. The method of measurement has been described in a previous memorandum ${ }^{3}$.' It is seen that as the length-to-width ratio becomes large - - an assumption of the theory - - the anti-resonant ratio approaches the value 3, whereas, the res onant ratio approaches a value somewhat above three. It thus seems that at least one piece of evidence supporting the theory has been found.

From these data, one can also get an idea of the length-to-width ratio which can be tolerated. The spread in the experimental data of the frequency ratio was about 0.01 . If one then looks for a value of the length-to-width ratio for which the ratio of the antiresonant frequencies for first and third harmonics deviate by more than .01 from the theoretical value, that is, less than 2.99 , then for values of the length-to-width ratio less than this, the effect of the finite length-to-width ratio has become apparent. This seems to be a ratio of about 7 or 8 . It is reasonable to assume that at this point where the effect of the finite length-to-width ratio becomes apparent, the error is almost entirely in the third harmonic measurement. Thus, since the effective length is only $1 / 3$ of the actual length in the third harmonic, a length-to-width ratio greater than about 2.5 is acceptable. That is, if only first harmonic frequencies are used, little error is introduced into the measurement by the finite length-to-width ratio providing

${ }^{3}$ 'Tech Memo 128-55-51, "A Study of the Effect of Temperature on the Ratio $\frac{d_{33}}{d_{31}}$ in Polycrystalline Barium Titanate", E. G. Franzak. 
that this ratio is 2.5 or greater. This is supported by the first harmonic data used in Figure 2, and is tabulated below. The agreement is as good as could be expected among elements of like dimensions.

\begin{tabular}{ccc} 
Element No. & Length/Width & \\
\hline 1 & 8.0 & \\
2 & 5.3 & 0.362 \\
3 & 5.3 & 0.358 \\
4 & 5.3 & 0.359 \\
5 & 4.0 & 0.369 \\
6 & 4.0 & 0.363 \\
7 & 4.0 & 0.356
\end{tabular}

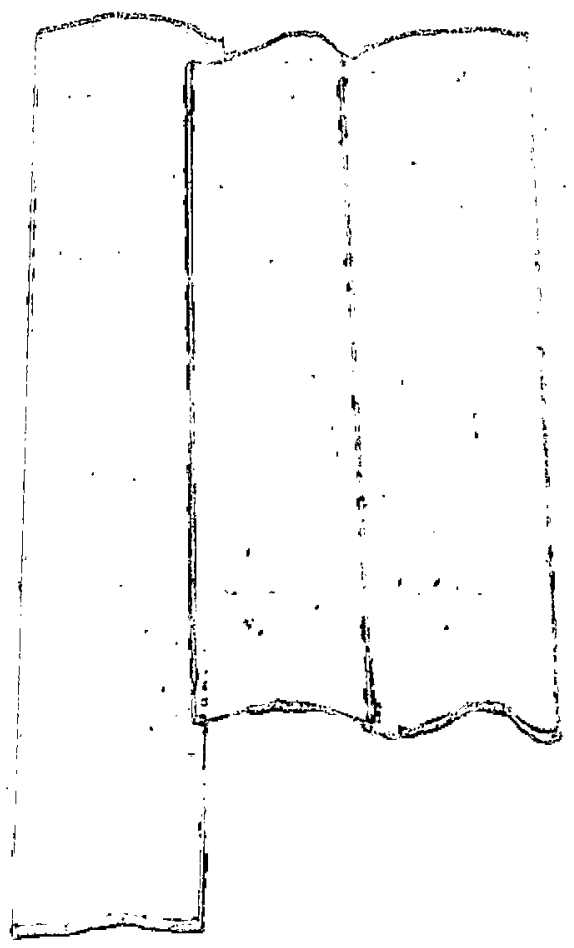

C. V. STEPHENSON- 5133

Case No. 590

July 29, 1955 
THIS PAGE

\section{WAS INTENTIONALLY LEFT BLANK}


